Bull. Austral. Math. Soc.

VOL. 40 (1989) [441-446]

\title{
ON AN EXTENSION OF A THEOREM OF SATO
}

LIAN-ZHONG YANG

Let $f(z)$ be a $n$-valued algebroid function of order $\lambda(0<\lambda<1)$, Sato obtained an elliptic theorem for $f(z)$ with a condition. In this paper, we prove that Sato's theorem is true without conditions and give a generalisation.

\section{INTRODUCTION}

Let $f(z)$ be a $n$-valued algebroid function defined by an irreducible equation

$$
A_{n}(z) f^{n}+A_{n-1}(z) f^{n-1}+\cdots+A_{1}(z) f+A_{0}(z)=0
$$

where $A_{j}(z)(j=0,1, \ldots, n)$ are entire functions without common zeros.

Let $T(r, f)$ be the characteristic function of $f(z)$ and $a(z)$ a rational function. Define

$$
\begin{gathered}
\delta(a, f)=1-\limsup _{r \rightarrow \infty} \frac{N(r, a, f)}{T(r, f)} \\
N(r, \infty, f)=N(r, f)
\end{gathered}
$$

With this notation, Sato [2] obtained the following result in 1981.

TheOREM A. Let $f(z)$ be a $n$-valued algebroid function of order $\lambda(0<\lambda<1)$, defined by the irreducible equation (1), and suppose that 0 is not a Valiron deficient value for $A_{n}(z)$. Let $a_{j}, j=1,2, \ldots, n$, be mutually distinct values, and put

$$
u_{j}=1-\delta\left(a_{n}, f\right) \text { and } \nu=1-\delta(\infty, f), 0 \leqslant u_{j}, \nu \leqslant 1
$$

Then there is at least one $a_{i}, 1 \leqslant i \leqslant n$, such that

$$
u_{i}^{2}+\nu^{2}-2 u_{i} \nu \cos \pi \lambda \geqslant n^{-2} \sin ^{2}(\pi \lambda) .
$$

If $u_{i}<n^{-1} \cos \pi \lambda$, then $\nu \geqslant 1 / n$; if $\nu<n^{-1} \cos \pi \lambda$, then $u_{i} \geqslant 1 / n$.

In this paper we shall prove that Sato's theorem is also true when $a_{j}(j=1,2, \ldots, n)$ are rational functions and without conditions.

Received 5 January 1989

Copyright Clearance Centre, Inc. Serial-fee code: 0004-9729/89 $\$ A 2.00+0.00$. 


\section{LEMMAS}

LEMMA 1. Let $f(z)$ be an $n$-valued transcendental algebroid function defined by equation (1), and set

$$
\begin{gathered}
A(z)=\max _{0 \leqslant i \leqslant n}\left|A_{i}(z)\right| \\
\mu(r, A)=\frac{1}{2 n \pi} \int_{0}^{2 \pi} \log A\left(\mathrm{re}^{i \theta}\right) d \theta
\end{gathered}
$$

Then

$$
|T(r, f)-\mu(r, A)|=O(1)
$$

Proof: See [3].

Leмma 2. Let $f(z)$ be a $n$-valued transcendental algebroid function defined by equation (1) and let $a_{i}(z)(i=1,2, \ldots, n)$ be rational functions, which are mutually distinct. Set

$$
\begin{aligned}
g_{i}(z) & =A_{n}(z) a_{i}^{n}+A_{n-1}(z) a_{i}^{n-1}+\cdots+A_{0}(z), i=1,2, \ldots, n, \\
g_{0}(z) & =A_{n}(z), g(z)=\max _{0 \leqslant i \leqslant n}\left|g_{i}(z)\right|, \quad\left(|z|=r>r_{0}\right), \\
\mu(r, g) & =\frac{1}{2 n \pi} \int_{0}^{2 \pi} \log g\left(\mathrm{re}^{i \theta}\right) d \theta .
\end{aligned}
$$

Then

$$
|\mu(r, g)-\mu(r, A)|=o(T(r, f)) .
$$

Proof: By the definition of a $\mu$-function

$$
\begin{aligned}
\mu(r, g)-\mu(r, A) & =\frac{1}{2 n \pi} \int_{0}^{2 \pi} \log \frac{\max _{0 \leqslant i \leqslant n}\left|g_{i}(z)\right|}{\max _{0 \leqslant i \leqslant n}\left|A_{i}(z)\right|} d \theta\left(z=\mathrm{re}^{i \theta}\right) \\
& \leqslant \frac{1}{2 n \pi} \int_{0}^{2 \pi} \log \sum_{i=0}^{n}\left(\left|a_{i}^{n}\right|+\left|a_{i}\right|^{n-1}+\cdots+1\right) d \theta \\
& =o(T(r, f)) \quad r \rightarrow \infty
\end{aligned}
$$

On the other hand, since

$$
\left(\begin{array}{c}
g_{0} \\
g_{1} \\
g_{2} \\
\vdots \\
g_{n}
\end{array}\right)=\left(\begin{array}{llllll}
1 & 0 & 0 & \ldots & 0 & 0 \\
a_{1}^{n} & a_{1}^{n-1} & a_{1}^{n-2} & \ldots & a_{1} & 1 \\
a_{2}^{n} & a_{2}^{n-1} & a_{2}^{n-2} & \ldots & a_{2} & 1 \\
\ldots \ldots \ldots \ldots \ldots \ldots \ldots \ldots \ldots \ldots & \ldots \ldots \\
a_{n}^{n} & a_{n}^{n-1} & a_{n}^{n-2} & \ldots & a_{n} & 1
\end{array}\right)\left(\begin{array}{l}
A_{n} \\
A_{n-1} \\
A_{n-2} \\
\vdots \\
A_{0}
\end{array}\right)
$$


and the coefficient determinant is not equal to zero $\left(|z|=r>r_{0}\right)$, we have

$$
A_{i}(z)=b_{i 0} g_{0}(z)+b_{i 1} g_{1}(z)+\cdots+b_{i n} g_{n}(z) \quad(0 \leqslant i \leqslant n)
$$

where the $b_{i j}$ are rational functions of the $a_{i}(0 \leqslant i \leqslant n)$.

Therefore, by the same reasoning, we have

$$
\mu(r, A)-\mu(r, g) \leqslant o(T(r, f))
$$

Lemma 2 is thus proved.

LEMMA 3. Let $g_{i}(z)(0 \leqslant i \leqslant n)$ be as defined in Lemma 2 , then

$$
T\left(r, g_{i} / g_{j}\right)-o(T(r, f)) \leqslant n T(r, f) \leqslant \sum_{i \neq j} T\left(r, g_{i} / g_{j}\right)+o(T(r, f))
$$

Proof: Let

$$
\begin{aligned}
U(z) & =\max _{0 \leqslant i \leqslant n}\left(\log \left|g_{i}(z)\right|\right) \\
U_{i j}(z) & =\max \left(\log \left|g_{i}(z)\right|, \log \left|g_{j}(z)\right|\right)
\end{aligned}
$$

Since

$$
U_{i j}(z)=\log ^{+}\left|g_{i} / g_{j}\right|+\log \left|g_{j}\right|
$$

we have

$$
\begin{aligned}
\mu(r, g) & =\frac{1}{2 n \pi} \int_{0}^{2 \pi} U\left(\mathrm{re}^{i \theta}\right) d \theta \\
& \geqslant \frac{1}{2 n \pi} \int_{0}^{2 \pi} U_{i j}\left(\mathrm{re}^{i \theta}\right) d \theta \\
& =\frac{1}{2 n \pi} \int_{0}^{2 \pi} \log ^{+}\left|g_{i} / g_{j}\right| d \theta+\frac{1}{2 n \pi} \int_{0}^{2 \pi} \log \left|g_{j}\right| d \theta \\
& =\frac{1}{n} m\left(r, g_{i} / g_{j}\right)+\frac{1}{n} N\left(r, 0, g_{j}\right)+o(T(r, f)) \\
& \geqslant \frac{1}{n} T\left(r, g_{i} / g_{j}\right)+o(T(r, f)) .
\end{aligned}
$$

By Lemmas 1 and 2, we have proved the left hand inequality. 
To prove the second inequality, we assume, without loss of generality, that $j=0$.

$$
\begin{aligned}
n \mu(r, g) & =\frac{1}{2 \pi} \int_{0}^{2 \pi} \log \max _{0 \leqslant i \leqslant n}\left|g_{i}(z)\right| d \theta \quad\left(z=\mathrm{re}^{\mathrm{i} \theta}\right) \\
& \leqslant \frac{1}{2 \pi} \int_{0}^{2 \pi}\left(\sum_{i=1}^{n} \log ^{+}\left|g_{i} / g_{j}\right|+\log \left|g_{0}\right|\right) d \theta \\
& =\sum_{i=1}^{n} m\left(r, g_{i} / g_{0}\right)+N\left(r, 0, g_{0}\right)+O(1) \\
& =\sum_{i=1}^{n} T\left(t, g_{i} / g_{0}\right)+N\left(r, 0, g_{0}\right)-\sum_{i=1}^{n} N\left(r, g_{i} / g_{0}\right)+O(1)
\end{aligned}
$$

Since (2) and $A_{j}(j=0,1, \cdots, n)$ have no common zeros, it follows that the common zeros of $g_{j}(j=0,1, \cdots, n)$ must be the zeros of the determinant

$$
D(z)=\left|\begin{array}{llllll}
1 & 0 & 0 & \ldots & 0 & 0 \\
a_{1}^{n} & a_{1}^{n-1} & a_{1}^{n-2} & \ldots & a_{1} & 1 \\
a_{2}^{n} & a_{2}^{n-1} & a_{2}^{n-2} & \ldots & a_{2} & 1 \\
\ldots \ldots \ldots \ldots \ldots \ldots \ldots \ldots \ldots \ldots \ldots & \ldots \\
a_{n}^{n} & a_{n}^{n-1} & a_{n}^{n-2} & \ldots & a_{n} & 1
\end{array}\right|(D(z) \not \equiv 0)
$$

By

$$
T(r, 1 / D(z))=o(T(r, f)), \quad r \rightarrow \infty
$$

we have

$$
N\left(r, 0, g_{0}\right) \leqslant \sum_{i=1}^{n} N\left(r, g_{i} / g_{0}\right)+o(T(r, f))
$$

Therefore

$$
n T(r, f) \sim n \mu(r, g) \leqslant \sum_{i=1}^{n} T\left(r, g_{i} / g_{0}\right)+o(T(r, f))
$$

this completes the proof.

\section{Results}

THeorem 1. Let $f(z)$ be a $n$-valued algebroid function of order $\lambda(0<\lambda<1)$, defined by the equation $(1), a_{j}(z)(j=0,1, \cdots, n)$ be $n$ mutually distinct rational functions. Put

$$
u_{j}=1-\delta\left(a_{j}, f\right), \quad \nu=1-\delta(\infty, f), \quad j=1,2, \cdots, n
$$


Then, there is at least one $a_{i}(1 \leqslant i \leqslant n)$ such that

$$
u_{i}^{2}+\nu^{2}-2 u_{i} \nu \cos \pi \lambda \geqslant n^{-2} \sin ^{2} \pi \lambda
$$

If $u_{i}<n^{-1} \cos \pi \lambda$, then $\nu \geqslant 1 / n$; if $\nu<n^{-1} \cos \pi \lambda$, then $u_{i} \geqslant 1 / n$.

Proof: Let $g_{i}(z)(i=0,1, \cdots, n)$ be defined in Lemma 2. By Lemma 2 the functions $g_{i} / g_{0}(i=1, \cdots, n)$ are of order at most $\lambda(0<\lambda<1)$. We use Edrei and Fuchs's idea [1] and their well-known representation. Then

$$
\begin{aligned}
T\left(r, g_{i} / g_{0}\right) \leqslant & \int_{0}^{\infty} N\left(t, 0, g_{i} / g_{0}\right) P\left(t, r, \beta_{i}\right) d t \\
& +\int_{0}^{\infty} N\left(t, g_{i} / g_{0}\right) P\left(t, r, \pi-\beta_{i}\right) d t
\end{aligned}
$$

where

$$
P(t, r, \alpha)=\frac{1}{\pi} \frac{r \sin \alpha}{t^{2}+2 r t \cos \alpha+r^{2}}, \quad(0<\alpha<\pi) .
$$

Since

$$
\begin{gathered}
N\left(r, 0, g_{i} / g_{0}\right) \leqslant N\left(r, 0, g_{i}\right)+o(T(r, f))=n N\left(r, a_{i}, f\right)+o(T(r, f)) \\
N\left(r, g_{i} / g_{0}\right) \leqslant N\left(r, 0, A_{n}\right)+o(T(r, f))=n N(r, f)+o(T(r, f)),
\end{gathered}
$$

therefore, by the definition of $u_{i}$ and $\nu$, given $\varepsilon>0$, there exists $t_{0}>0$, such that for $t \geqslant t_{0}$

$$
\begin{aligned}
& N\left(t, 0, g_{i} / g_{0}\right) \leqslant n\left(u_{i}+\varepsilon\right) T(t, f) \quad(1 \leqslant i \leqslant n) \\
& N\left(t, g_{i} / g_{0}\right) \leqslant n(\nu+\varepsilon) T(t, f) .
\end{aligned}
$$

By Lemma 3 and (3), we have

$$
\begin{aligned}
T(r, f) & \leqslant \sum_{i=1}^{n} \int_{t_{0}}^{\infty}\left(u_{i}+\varepsilon\right) T(t, f) P\left(t, r, \beta_{i}\right) d t \\
& +\sum_{i=1}^{n} \int_{t_{0}}^{\infty}(\nu+\varepsilon) T(t, f) P\left(t, r, \pi-\beta_{i}\right) d t+o(T(r, f)) .
\end{aligned}
$$

Using this inequality and by adopting the arguments used by Sato [2] or Edrei and Fuchs [1], Theorem 1 follows.

Corollary. If 0 is a Valiron deficient value for $A_{n}(z)$, Theorem $A$ is also true. 


\section{ReFERENCES}

[1] A. Edrei and W.II. Fuchs, 'The deficiencies of ineromorphic functions of order less than one', Duke Math. J. 27 (1960), 233-249.

[2] T. Sato, 'Remarks on the deficiencies of algebroid functions of finite order', Proc. Japan Acad. Ser. A Math. Sci. 57 (1981), 101-105.

[3] G. Valiron, 'Sur la derivee des fonctions algebroides', Bull. Soc. Math. 68 (1931), 17-39.

Department of Mathematics

Shandong University

Jiuan

China 